\title{
Design and Implementation of Application for the Hearing Impaired People
}

\author{
ChoulWoo Kim ${ }^{1}$, Dongyun Kim ${ }^{1}$, Hyundo Lee ${ }^{1}$, Hanseong Park ${ }^{1}$, Giung Kwon ${ }^{1}$, Eunju Park $^{1}$, Hankyu Lim ${ }^{1, *}$ \\ ${ }^{1}$ Dept of Multimedia Engineering, Andong National University, Andong, Republic of Korea
}

\begin{abstract}
With the development of various information and communication technologies, numerous applications are being developed and used in various fields in this modern society. However, these applications are mostly designed to provide convenience to general users, and applications for people with disabilities are small in number and inconvenient to use. Therefore, this study designed and implemented an application for the hearing impaired in particular. This application is intended to enable users with hearing impairment to communicate more easily with other people and to provide convenience in everyday life and emergency situations. We believe that many applications should be developed in various fields to provide the convenience of life to those who are experiencing information gap due to disability.
\end{abstract}

\section{Introduction}

The number of people with disabilities is increasing and the occurrence rate of hearing impairment among the disabilities is the third highest after physical disability and visual impairment [1]. According to the Ministry of Health and Welfare's '2014 Survey on the Status of People with Disabilities', the number of hearingimpaired people in South Korea was approximately 310,000 in 2014. Most of the people with hearing impairment acquired the disability and the higher the age, the higher the incidence became [2]. Among the various applications of information and communication technology, smart devices have become an essential part of everyday life such as educational and administrative services, shopping, and banking [3]. Applications in various fields are being developed for the increasing number of smartphone users and user-oriented smartphone applications for user convenience are also increasing [4]. However, smartphones are not convenient communication tools for people with hearing impairment, who have difficulty using even the most basic voice calls on a smartphone [5]. Even though there are some applications that can be used by people with disabilities who have limited physical and cognitive functions through education and training, the number and types of applications for the disabled in South Korea are very limited compared to other countries [3]. Furthermore, even the applications developed for the hearing-impaired are inconvenient to use because their services do not consider the hearing-impaired users properly [6].

Consequently, this study designed and developed an application for the hearing impaired people who need special functions that are not provided by most of the applications made for general users. In order to develop this application, we examined the lacking features of existing applications for the hearing-impaired and investigated the daily lives of hearing-impaired people to find and improve their inconveniences. The application developed in this study implemented a feature to easily communicate through the sign language to solve the daily discomfort of the hearing impaired people, SOS feature to alert an emergency situation, a feature to set alarms by vibration to alert the hearing impaired people, and a feature to search the voices of non-disabled people and communicate them by sign language. Moreover, we added other features to help the hearing impaired such as food ordering and guidance to nearby welfare facilities for the hearing impaired. This application was implemented using Android Studio to allow the hearing impaired to use it more conveniently with various smart devices.

One limitation of this study is that it may be less usable for the disabled because people who responded to the questionnaire survey to investigate the feasibility of application development were non-disabled people. The usability of this application will be improved in our follow-up study.

The application developed in this paper is expected resolve the information gap and improve the convenience of living for the disabled people.

\section{Related Research}

\subsection{Hearing impairment}

Hearing impairment refers to a disorder in the ability to hear due to a damage of the hearing organ. Hearing impairment varies widely according to the severity from mild hearing loss, which cannot hear small sounds, to

* Corresponding author: hklim@anu.ac.kr

(C) The Authors, published by EDP Sciences. This is an open access article distributed under the terms of the Creative Commons Attribution License 4.0 (http://creativecommons.org/licenses/by/4.0/). 
severe hearing loss, which cannot hear loud sounds [1]. Whereas the non-disabled people communicate by speech, the hearing impaired communicates in a different language according to their degree of disability.

There are various communication methods for the hearing impaired, such as sign language, oral method, total communication, and bilingual approach [7].

\subsection{Android Studio}

Android is a software stack and an operating system for smartphones and other mobile devices, which includes middleware, user interfaces, and standard applications [8]. Android allows developers to write applications in Java language and provides runtime library for running complied byte codes. Furthermore, they provide various tools and APIs required to develop applications through the Android Software Development Kit (SDK). Android Studio is an Android development tool based on IntelliJ IDEX which is a famous integrated development environment (IDE). Therefore, developers can easily use various functions provided by IntelliJ.

In this study, we developed an application using Android Studio and created a virtual machine through the emulator which is a feature of Android Studio to implement it in an environment that is identical to the actual mobile phone.

\section{Requirement Analysis}

To develop the app for the convenience of the hearing impaired in this study, we added and improved various functions by conducting a questionnaire survey and benchmarking.

\subsection{Survey Investigation}

The survey was conducted between October 1 and 6, 216 using Naver Form. The number of respondents was 96 in total, consisting of 73 males and 23 females. Figure 1 to 4 show the graphs of significant results obtained from the survey.

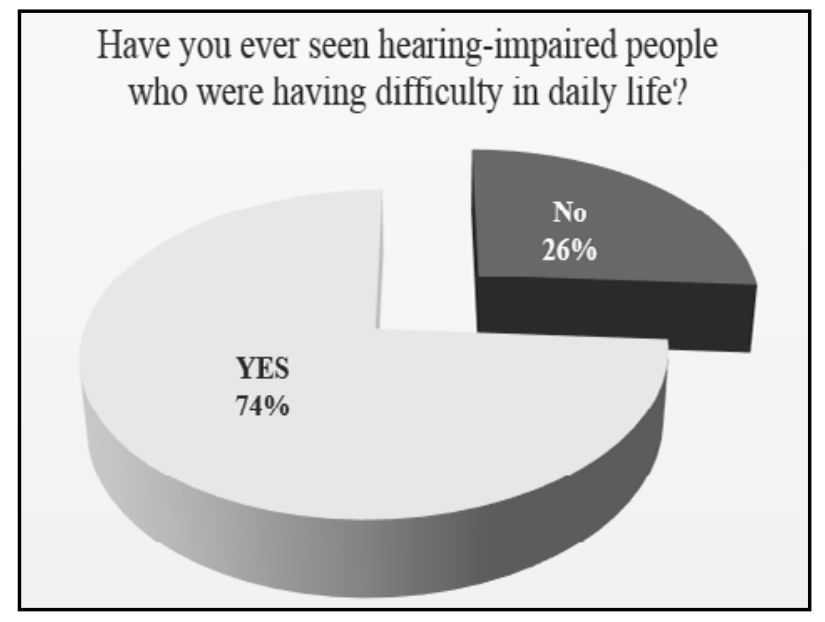

Fig. 1. Survey Result 1

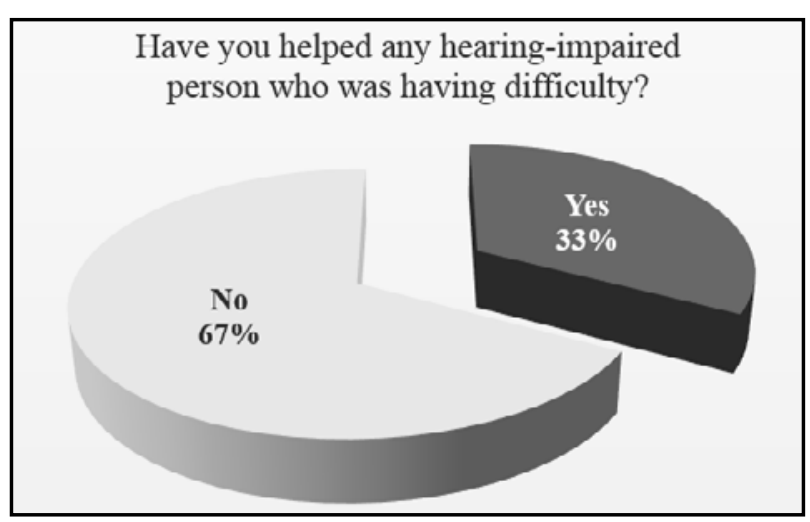

Fig. 2. Survey Result 2

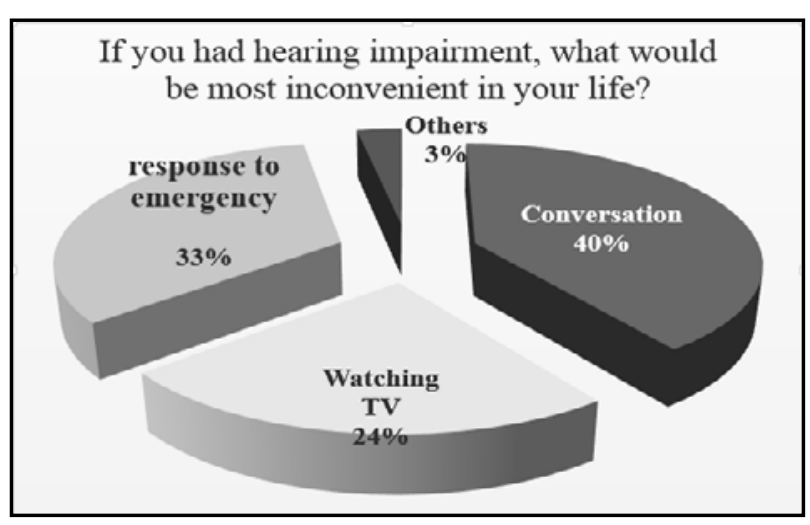

Fig. 3. Survey Result 3

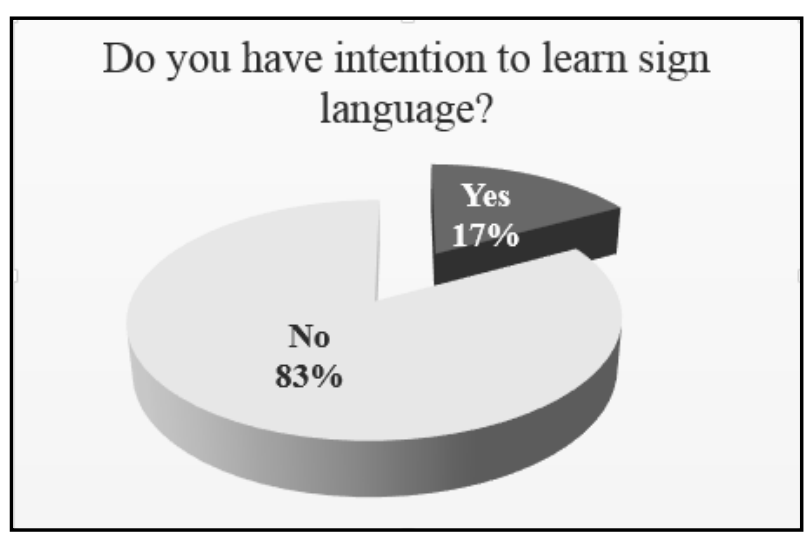

Fig. 4. Survey Result 4

As a result of the survey, $74 \%$ of the respondents answered yes to "I have seen hearing-impaired people who were having difficulty in daily life." To the question, "Have you helped any hearing-impaired person who was having difficulty?" $67 \%$ answered that they have not. To the question, "If you had hearing impairment, what would be most inconvenient in your life?" the largest number of respondents answered 'conversation,' followed by 'response to emergency,' 'watching TV,' and 'Others.' Furthermore, $83 \%$ of the respondents answered that they have no intention to learn sign language.

The survey results showed that many respondents have seen hearing impaired people but did not help them. Many respondents thought that conversation would be the most difficult if they had hearing impairment. In 
addition, most of the respondents had no intention to learn sign language for communication with the hearing impaired people. Therefore, the survey results suggest that information and communication technology and other helpful devices are needed for communication between the non-disabled and the hearing impaired.

\subsection{Benchmarking}

To improve the usefulness of the application developed in this study, the currently used applications for the hearing impaired were investigated for benchmarking and their weak points were noted for supplementation. Figure 5 shows the benchmarked applications.

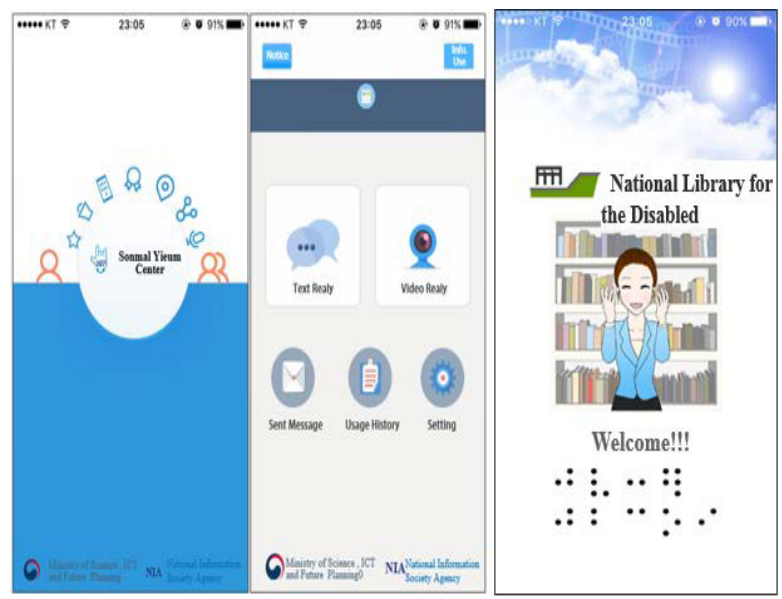

Fig. 5. 'Sonmal Yieum Center' and 'National Library Video Data Pavilion for the Disabled'

As a result of benchmarking, 'Sonmal Yieum Center' helped communication between hearing impaired people and relay person by translating or converting sign language into texts. 'Share Typing' provides real-time subtitles and stenographers delivered messages through real-time typing at schools, churches, seminars, etc. 'National Library Video Data Pavilion for the Disabled' provided sign language and subtitles to allow the hearing impaired to read library materials anywhere. The benchmarking results showed that applications to help the daily life of the disabled were insufficient and the translation feature using sign language was difficult to use because it required a relay person. Therefore, the application for the hearing impaired developed in this study added a feature to search dialogues and information for general users as well as for the hearing impaired during conversations where the hearing impaired feel most discomfort. Sign language dictionary, sign language search and browsing, and expert connection features were added to design and implement a more convenient and fast application.

\section{Design}

\subsection{Flowchart}

Figure 6 shows the flowchart of the application developed in this study. The numerical word search, voice translation, vibration alarm, SOS, substitute order, and welfare facility search features were defined.

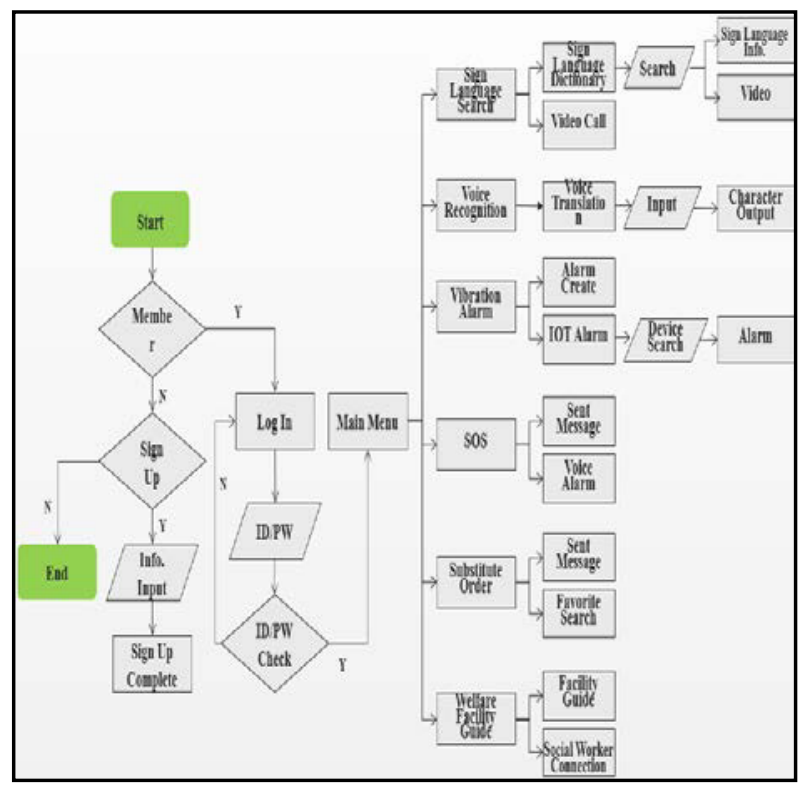

Fig. 6. Flowchart of the Application

\subsection{UI Design}

The following shows the user interface (UI) of the application developed in this study. The image at left in Figure 7 is the 'Log In' UI for logging in to or signing up for the application, which consists of password input field, sign up and log in buttons. The image at right in Figure 7 shows a page where you can choose any function of the application to run the function.

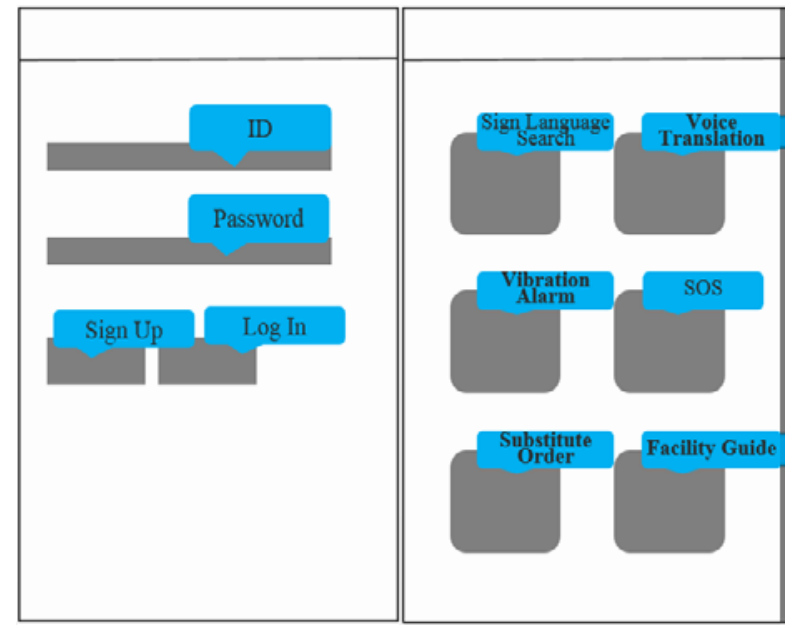

Fig. 7. 'Log In' and 'Main Screen' UI

The UI page for 'sign language search' is shown at left in Figure 8. It consists of a sign language dictionary where users can search sign language by connecting with a sign language search site and a feature for making a video call with a sign language expert.

The UI at right in Figure 8 shows a page that can be used by the hearing impaired in an emergency or to seek help from surrounding persons. It includes messaging feature for sending text messages to the stored phone 
number and alarm feature to notify emergency to other people in the vicinity by large siren sound.

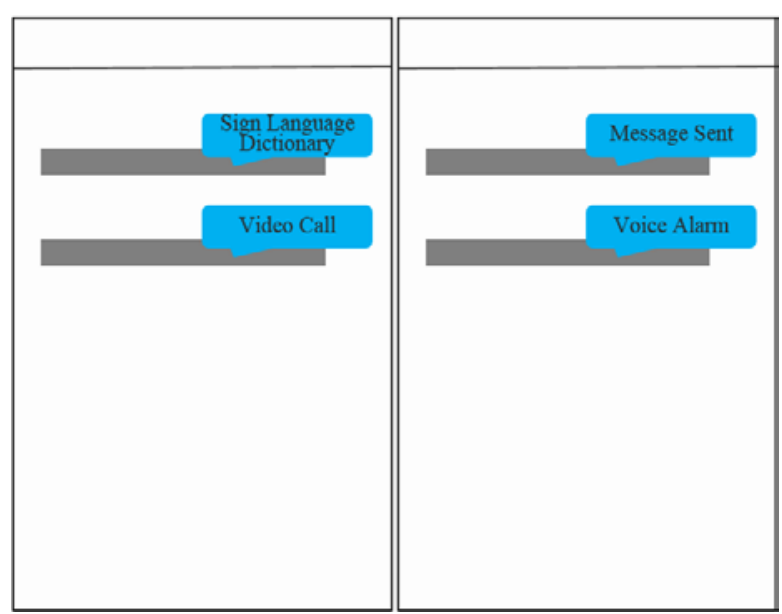

Fig. 8. 'Sign Language Search' and 'SOS Page' UI

\section{Implementation}

In this study, eight functions were implemented as prototype, including $\log$ in, sign up, sign language search, void translation, vibration alarm, SOS, substitute order, and welfare facility guide.

\subsection{Log In and Sign Up}

Figure 9 below shows the screens for Log In and Sign Up. The application developed in this study was designed to ask for the minimum personal information, considering that the users are disabled people who have difficulty using the application When the ID that a user wants is already taken by another user, a message requesting recently is displayed. When user clicks the JOIN button, the sign-up process is completed and the Log In page appears. When user clicks the 'CANCEL' button, the main page appears.

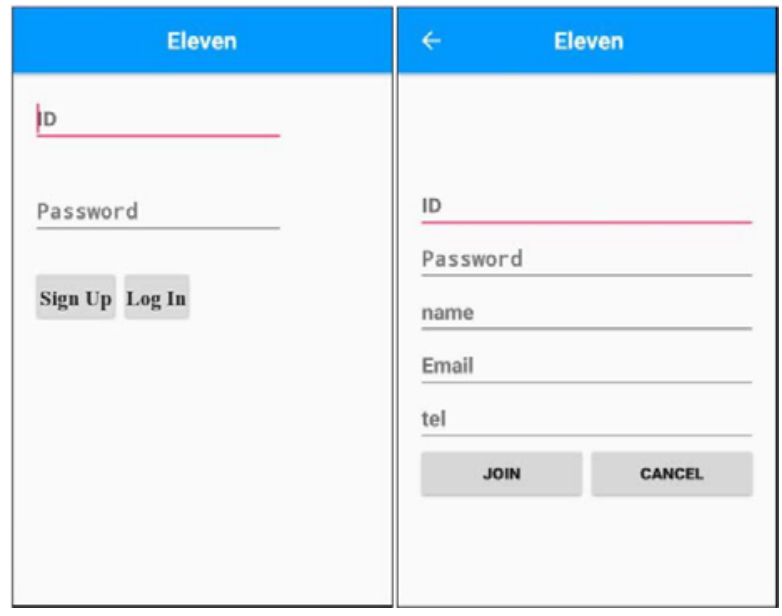

Fig. 9. 'Log In' and 'Sign Up' Implementation screen

\subsection{Intro and Main Screen}

Figure 10 below shows the intro and main screens of the application. The intro screen was named Eleven (11) by rotating the equal sign ' $=$ ' which means equality by 90 degrees. When the main screen appears after log in, the menu icons appear, including sign language search, voice translation, vibration alarm, SOS, substitute order, and welfare facility guide. Users can click an icon to go to the desired menu.

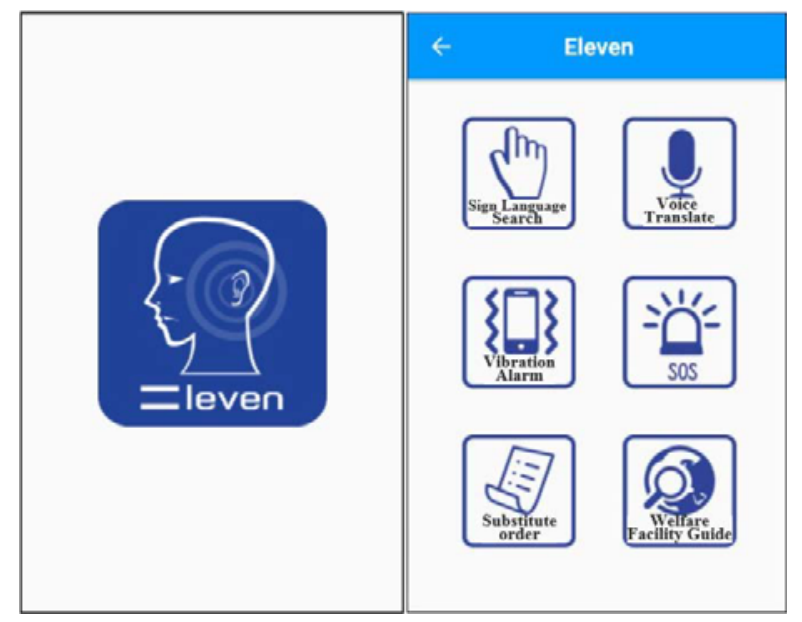

Fig. 10. 'Intro' and 'Main' Implementation Screen

\subsection{Sign Language Search and Sign Language Dictionary}

Figure 11 below shows the sign language search screen. This screen appears when the sign language search icon is clicked in the main screen. The sign language dictionary screen shows the Korean Sign Language Dictionary page where you can enter a sign language to search and play videos.

\begin{tabular}{|c|c|}
\hline$\leftarrow$ & Eleven \\
\hline Sign Language Dictionary & \multirow{3}{*}{$\begin{array}{l}\text { Now, Science experience in Koraan sign languga } \\
\text { Korean Sign Language } \\
\text { Dictionary } \\
\text { Fine Detail } \odot \text { Telling }\end{array}$} \\
\hline \multirow[t]{5}{*}{ Video Call } & \\
\hline & \\
\hline & $\cdots$ Notice \\
\hline & Another Language \\
\hline & E Popular Sign L.anguage \\
\hline
\end{tabular}

Fig. 11. 'Sign Language Search Screen' and 'Sign Language Dictionary Screen' Implementation

\subsection{Voice Translation Recognition}

Figure 12 below shows the voice translation recognition screen. When you click the mic button and say words, they are recognized and displayed as texts in TextView. 


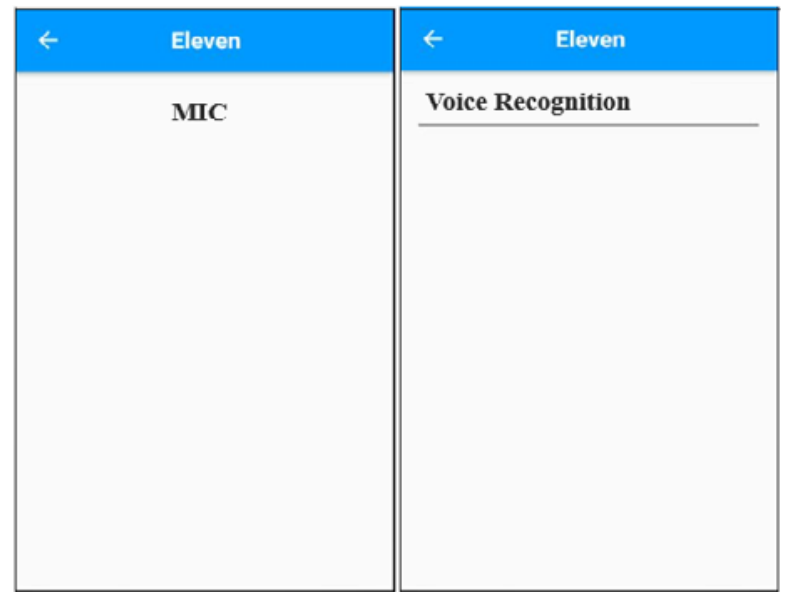

Fig. 12. 'Voice Recognition Screen' Implementation

\subsection{Vibration Alarm and Alarm Generation screen}

Figure 13 below shows the vibration alarm and alarm generation screens. This screen appears when you click the vibration alarm icon in the main screen. When you select a date and time and click the SET button in the alarm generation screen, the alarm goes off by vibration at the selected time.

\begin{tabular}{|c|c|c|c|}
\hline$\leftarrow$ & $\leftarrow$ & Eleven & \\
\hline Alarm Generation & & & \\
\hline \multirow[t]{7}{*}{ IOT Alarm } & Oct & 26 & 2015 \\
\hline & Nov & 27 & 2016 \\
\hline & Dec & 28 & 2017 \\
\hline & 2 & 15 & AM \\
\hline & 3 & 16 & PM \\
\hline & 4 & 17 & \\
\hline & & SET & RESET \\
\hline
\end{tabular}

Fig. 13. 'Vibration Alarm' and 'Alarm Generation Screen' Implementation

\subsection{SOS and Substitute Order}

Figure 14 below shows the SOS screen. A preset text message is sent to the pre-set number in an emergency. When you select the siren icon, the emergency is notified to people in the vicinity by outputting a siren sound. When you click the siren button once again, the siren sound will stop.

Figure 15 below shows the substitute order screen. When you click the 'Substitute Order' button, select the connected restaurant phone number and order item, and click the Order button, a text message is sent to the restaurant.

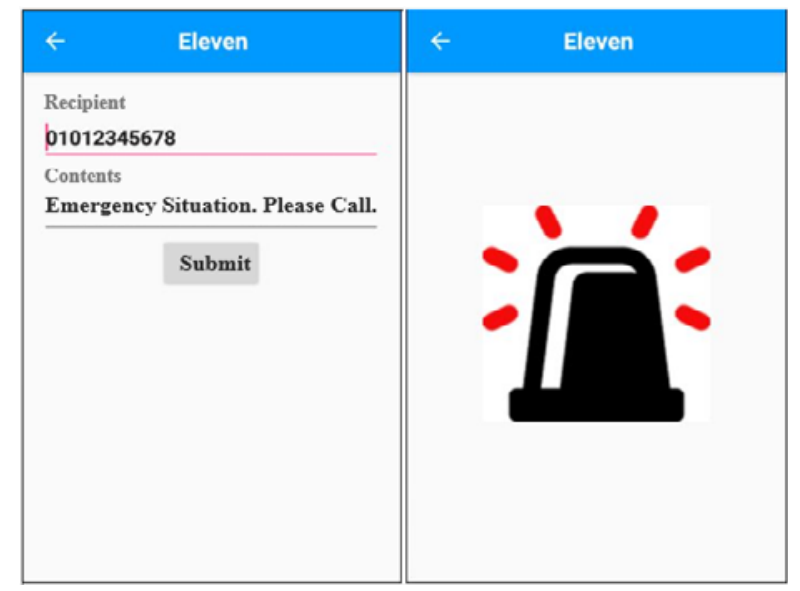

Fig. 14. 'SOS Screen' and 'Sound Alarm Screen' Implementat ion

\begin{tabular}{|l||l|}
\hline \multicolumn{1}{|c|}{ Eleven } & $\leftarrow \quad$ Eleven \\
\hline Message Sent & $\begin{array}{l}\text { Number } \\
\text { pavorite Search }\end{array}$ \\
$\begin{array}{l}\text { Order Contents } \\
\text { Hot Fried 1, } \\
\text { Credit Card }\end{array}$ \\
\hline \\
\hline
\end{tabular}

Fig. 6. 'Substitute Order Screen' and 'Message Sent Screen' I mplementation

\subsection{Welfare Facility Guide}

Figure 16 below shows the welfare facility guide screen. When you click the Facility Guide or Social Worker Connection button, the website of Korean Association of the Welfare Institutes for the Disabled appears.

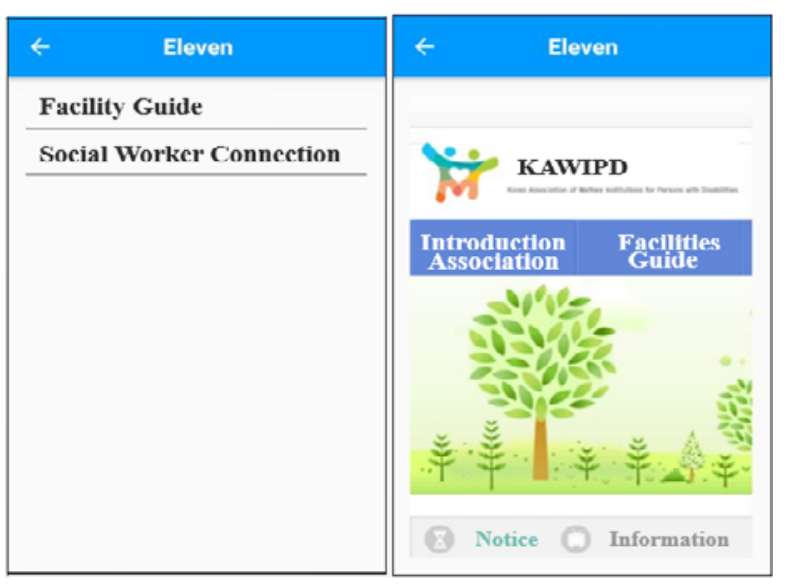

Fig. 7. 'Welfare Facility Guide Screen' Implementation

\section{Conclusion}


An application for the smooth communication and convenient life of the hearing impaired people was designed and implemented. For this application, searching sign language search feature and voice to text translation feature were designed to facilitate communication between the hearing impaired and nondisabled people. In addition, vibration alarm and SOS alert features were designed and implemented for the safety of the hearing impaired. In addition, welfare facility guide feature was added to help users easily find welfare facilities.

In South Korea, smartphones have become popular among the disabled, but there are not many applications that have considered the environment for the disabled. This study intended to help the hearing impaired people to lead more convenient and safe life and to communicate easily with non-disabled people. This application will help bridge the information and communication gap of the disabled.

The development and propagation of smartphone applications for the disabled are not as active as those for general users. However, more smartphone applications for the disabled should be developed because they have constant demand. Furthermore, many applications in various areas are required to resolve the information gap and improve the convenience of living for people with various disabilities.

\section{Acknowledgment}

"This research was supported by the MSIP(Ministry of Science, ICT and Future Planning), Korea, under the Seoul Accord Vitalization Program(IITP-2017-2011 0 00559) supervised by the IITP(Institute for Information \& communications Technology Promotion)"

\section{References}

[1] E. Jang, B. Kim, "The study on the GUI guideline in the survey of deaf needs. - Focused on municipal seodaemun deaf community welfare center.," Design Convergence Study 43, Vol. 12, pp, 214 217, No. 6 (2013)

[2] Ministry of Health and Welfare's, "2014 Survey on the Status of People with Disabilities," Ministry of Health and Welfare's, pp. 149-154 (2015)

[3]

http://www.ablenews.co.kr/News/NewsContent.aspx ?CategoryCode $=0002 \&$ NewsCode $=0034201303200$ 93247143741 (03, 2017)

[4] J. Oh, B. An, "Android Based Mobile Smart Alarm," The Journal of the Institute of Internet, Broadcasting and Communication, Vol, 12, No. 3, pp. 141-147 (2012)

[5] J. Kim, "Research studies on user's needs on screen mobile phone for people with hearing disorder," The Graduate School of Design Ewha Womans University, pp. 14-20 (2008)

[6] K. Kim, "A design guideline to develop an application for cultural facility in consideration of the deaf," Graduate School of Industry and Engineering Seoul National University of Science and Technology, pp. 7-12 (2014)

[7] S. Woon, Kyungjin Kim, Il Heo, "The Study on Proposal to Apply Sign Language in the Field of Education for Deaf Students," The Journal of Special Education: Theory and Practice, Vol. 14, No. 4, pp. 113-132 (2013)

[8] S. Kim, "Android programming complete guide 1," Hanbit Media (2016) 